\title{
Endoskopieturm, Einstellung, Pflege, Dokumentation
}

\author{
Werner Koithan
}

\section{Zusammenfassung}

Seit der Erstveröffentlichung von Bircher im Jahr 1921 hat die Arthroskopie durch Verbesserung der Technik enorme Fortschritte gemacht. Dies gilt für die arthroskopische Optik, die heute für verschiedene Gelenke zwischen 1 und $4 \mathrm{~mm}$ variieren kann. Xenon-Lichtquellen sind leistungsstark mit einer Farbtemperatur von $6000^{\circ}$ Kelvin. Unter den Lichtkabeln haben sich die Fiberglaskabel im Wesentlichen durchgesetzt, während Fluidkabel für spezielle Einsätze mit möglichst originalgetreuer Wiedergabe der Gelenkstrukturen vorbehalten bleiben. Unter den Kameras sind die Ein- und Dreichip-Kameras sehr verbreitet. Die neuen Entwicklungen gehen auch hier in Richtung Digitalkameras, die auf dem CCD-System beruhen. Damit ist auch die Auf- bereitung der Daten in verschiedenen Formaten möglich. Unter den Spülsystemen haben sich 2 Systeme durchgesetzt: Einmal das Pumpensystem über die Rollenpumpe oder die Auffüllung mittels Schwerkraft. Bei den Lagerungen am Schultergelenk hat sich die Seitenlagerung und die BeachChair-Position in gleicher Weise durchgesetzt. Die Beach-Chair-Position sollte insbesondere dann gewählt werden, wenn im Anschluss an einen arthroskopischen Eingriff eine offene Rekonstruktion der Rotatorenmanschette oder ventralen Kapsel geplant ist. Die wichtigste Dokumentationsform ist natürlich der Operationsbericht. Daneben haben sich Dokumentationsbögen durchgesetzt und in neuerer Zeit die digitalen Standbildrekorder, mit denen die Speicherung und Archivierung der Bildinformation möglich ist.

\section{Arthroskopie}

\section{Historie}

Unabhängig voneinander begann die Entwicklung der Arthroskopie durch Bircher in Aarau, durch Burmann in New York und durch Tagaki und Watanabe in Tokio. Die Erstveröffentlichung der Arthroskopie kam 1921 von Bircher (Abb. 1). Burmann, Finkelstein und Meyer (1936) aus dem Hospital for Joint Deseases publizierten in den 30er-Jahren zahlreiche klinische und experimentelle Arbeiten über die Arthroskopie verschiedener Gelenke. Eine Schule der Arthroskopie wurde 1918 von Tagaki in Tokio gegründet und von Wata-

OP-JOURNAL 2005; 21: 38-44

(c) Georg Thieme Verlag KG Stuttgart · New York nabe fortgesetzt. Der erste Arthroskopieatlas Watanabes erschien 1957. Zwischen 1930 und 1960 entstanden 21 verschiedene Arthroskope, deren spezifische Modifikation den jeweiligen technischen Möglichkeiten und den steigenden Anforderungen, z.B. der Farbphotographie, entsprachen. 1960 entstand das berühmte Instrument Watanabe Nr. 21, das mit einem Lämpchen an der Spitze heute zwar vorsintflutlich anmutet, welches aber entscheidend zur weiteren Entwicklung und weltweiten Verbreitung der Arthroskopie beitrug. Neue technische Entwicklungen mit Kaltlicht und Glasfaserleitung, deren Technik auf der Entdeckung Fourestiers aus dem Jahr 1952 basierte mit dem Prinzip der Lichtleitung durch lange Quarzstäbe. Dadurch wurde die Wolframbirne an der Gerätespitze abgelöst, die Lichtquelle - ein Kaltlichtprojektor - rückte außerhalb des Instrumentes. Nach der Änderung der Lichtquelle erfährt die Entwick- lung einen weiteren Schub durch die Einführung der Stablinsentechnik nach Hopkins (KST). Durch Änderung des Luftgemisches zwischen den Linsen und der zylindrischen Linsenform erhält man beim Hopkins-System reflexionsärmere Bilder bei erheblich erweitertem Gesichtsfeld und erhöhter Lichtintensität. Eine Verminderung der Streustrahlung, eine Verbesserung der Farbqualität und ein höheres Auflösungsvermögen ließen es zu, dass bei guter Bildqualität der Durchmesser der Instrumente vermindert werden konnte. Neben der guten Bildqualität wurde die Arthroskopie jetzt auch videogerecht [3].

\section{Arthroskopische Optik}

Wesentlicher Bestandteil des arthroskopischen Instrumentariums ist die Optik, die aus einem Okularteil, dem Lichtkabelansatz, den Linsen sowie Fasern für die Lichtleitung besteht. Linsen, Lichtleitfasern und Metallummantelung bilden den Schaft der Optik (Abb. 2).

Die heutigen Optiken basieren auf dem Hopkins-Stablinsensystem und ermöglichen damit ein wesentlich größeres Blickfeld bei gleichzeitig kleinerem Gesamtdurchmesser. Gleichfalls ist mit den Hopkins-Optiken eine größere Bildhelligkeit verbunden.

Die Optiken werden in verschiedenen Schaftlängen angeboten, wobei sich für das Kniegelenk eine Schaftlänge von $18 \mathrm{~cm}$ bewährt hat. Kürzere Optiken haben den Nachteil, dass bei großen Kniegelenken z.B. die Refixation des Innenmeniskus erschwert ist. Die Optiken werden in verschiedenen Durchmessern angeboten. Der Durchmesser des Optikschaftes setzt sich aus dem Linsensystem (Stablinsen, Glasfasern zur Lichtleitung und Metallummantelung) zusammen. Die Durchmesser der gebräuchlichen Optiken für die Arthroskopie verschiedener Gelenke liegen zwischen 1 und $4 \mathrm{~mm}$. Für das Kniegelenk hat sich die $4 \mathrm{~mm} 30^{\circ} \mathrm{Op}$ - 


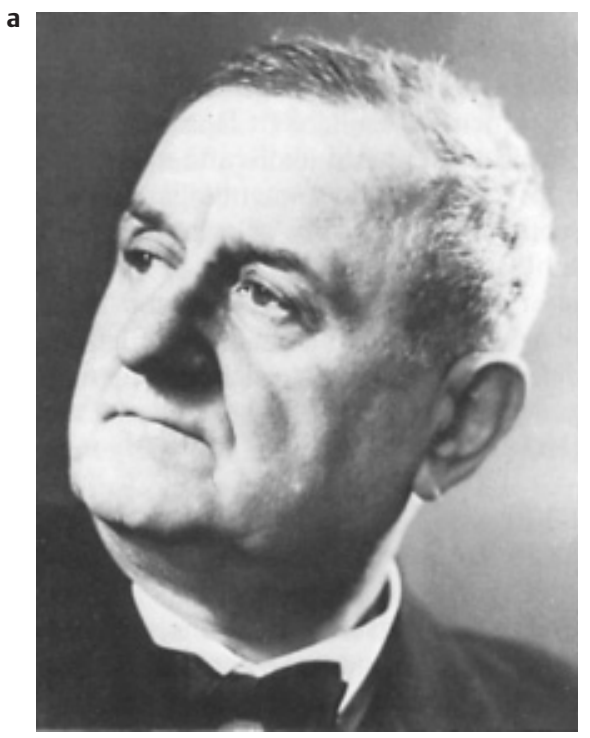

tik durchgesetzt. Kleinere Optiken werden für Sprung- und Handgelenk verwendet.

\section{Sterilisierbarkeit}

In früheren Zeiten waren Optiken nur gassterilisierbar oder einzulegen. Dies ist aus Gründen der Gasbelastung (Umweltproblem) heute abzulehnen, sodass Optiken dampfsterilisierbar sein sollten $\left(134^{\circ}\right)$. Mit dem Einlegen der Optiken in eine Desinfektionslösung ist keine ausreichende Keimfreiheit zu erzielen.

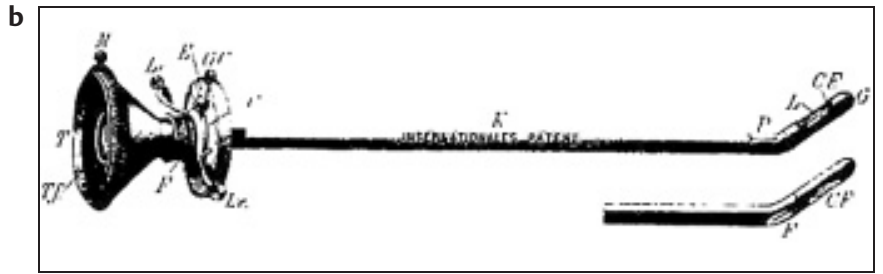

Abb.1 (a) Eugen Bircher veröffentlichte 1921 erstmals eine Arbeit über 18 Arthro-Endoskopien an Patienten. (b) Zystoskop nach Leiter, welches für die ersten Arthroskopieversuche am Knie verwendet wurde (aus Donner 1979).

\section{Alterungsprozess}

Auch Optiken unterliegen einem physiologischen Alterungsprozess. Je häufiger eine arthroskopische Optik verwendet wird, desto schneller altert sie. Der Alterungsprozess wird beschleunigt durch häufiges Blitzsterilisieren und gleichzeitiges schnelles Wiederabkühlen der empfindlichen Optiken.

Alterungserscheinungen machen sich durch ein trübes, lichtschwaches Bild bemerkbar. Als Kontrolle ist es hilfreich, an die unsterile Optik ein Lichtkabel und die Kamera anzuschließen und dann z. B. die eigene Handinnenfläche auf dem Monitor zu betrachten.

Zeigen bei Verwendung eines guten Lichtkabels und nach Säuberung von Kameraobjektiv, Okular und Optikspitze im-

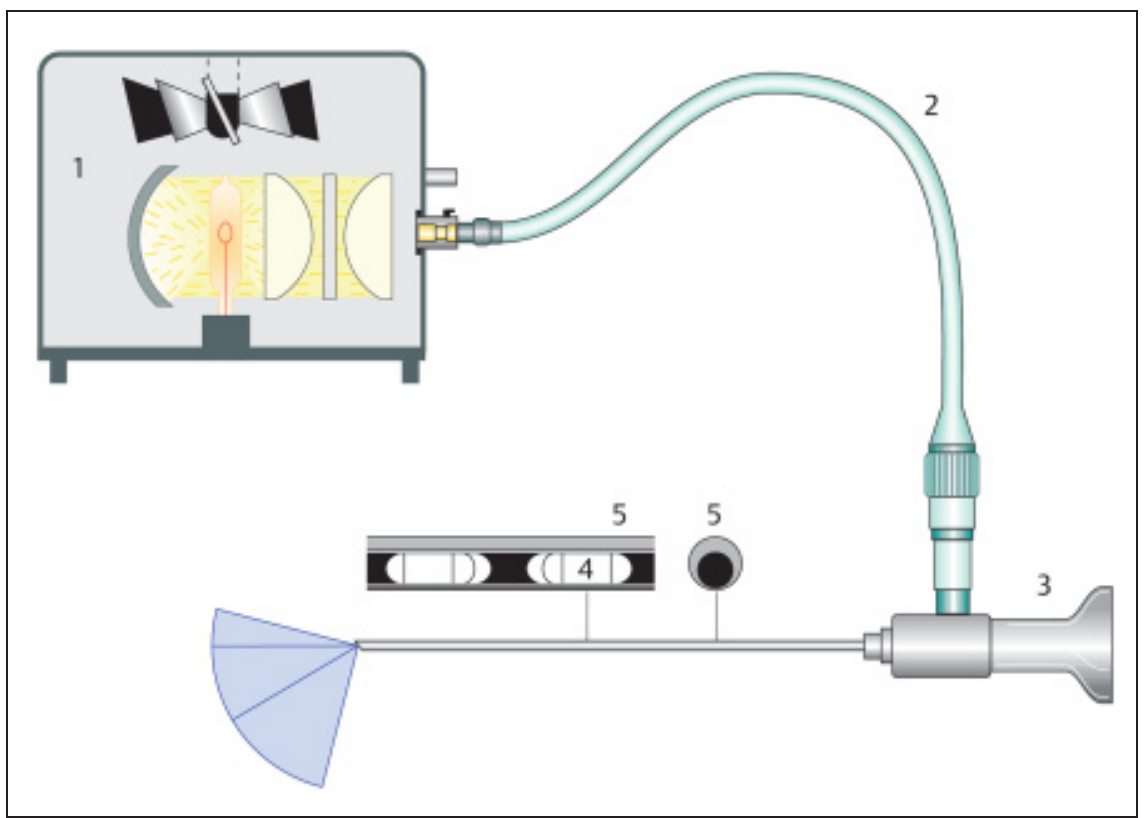

Abb.2 Schema einer Optik mit angeschlossenem Lichtsystem: Lichtquelle (1), Lichtleitkabel (2), $30^{\circ}$-Weitwinkeloptik mit Okular (3). Der Schaft der Optik enthält Linsensysteme (4) und Glasfaserbündel zur Lichtleitung (5) (Fa. Arthrex). mer noch stumpfe, unscharfe Areale oder ist die gesamte Optik trüb, kann man versuchen, das optische Ergebnis durch Erhöhung der Intensität der Lichtquelle zu verbessern (Autoshutter regelt ab). Ist dies nicht möglich, sollte die Optik zur Reparatur gesandt werden. Auch direkte Beschädigungen der Optikspitze durch mechanische oder motorisierte Instrumente führen zu einer reduzierten Bildqualität, die sich in lokalisierten Kratzern oder Bildtrübungen zeigt. Besonders gefährlich für die Optikspitze ist die Verwendung des Lasers. Hierbei kann es zu einer erheblichen Schädigung der Linsen an der Optikspitze kommen $[3,4]$.

\section{Lichtquelle}

Unterschieden werden Kaltlicht- und Xenon-Lichtquellen (Abb. 2).

Das Licht wird über ein Lichtleitkabel und die in der arthroskopischen Optik integrierten Glasfasern in das Gelenk geleitet.

\section{Kaltlichtquelle}

Verschieden starke Kaltlichtquellen werden angeboten. Als Minimalleistung sollten 150 Watt zur Verfügung stehen. Eine höhere Leistung bedeutet eine Reservefunktion, wenn z.B. Gelenkareale mit schlechten reflektierenden Flächen inspi-

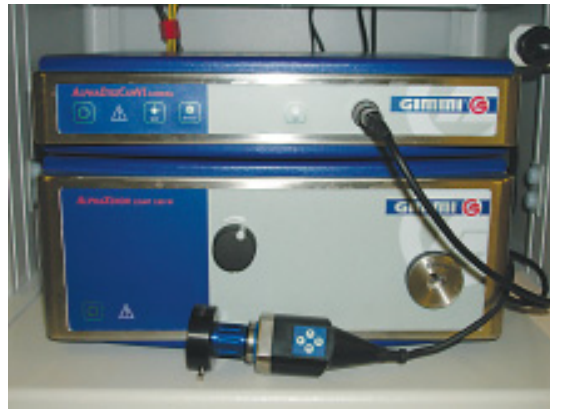

Abb. 3 Xenon-Lichtquelle und Steuereinheit mit Kamerakabel und Kamera. 
ziert werden müssen oder die Optik etwas a getrübt oder das Lichtkabel etwas älter ist.

\section{Xenon-Lichtquelle}

Die Xenon-Lichtquellen (Abb. 3) sind leistungsstärker und entsprechen mit einer Farbtemperatur von $6000^{\circ}$ Kelvin eher dem Tageslicht als Kaltlichtquellen (Farbtemperatur von $3400^{\circ}$ Kelvin). XenonLichtquellen sind dann angezeigt, wenn es auf eine möglichst hohe Farbqualität, z.B. für die Videodokumentation, zur Dokumentation arthroskopischer Befunde (digitale Dokumentation oder Videoprints) ankommt. Die Lichtquelle wird erst dann eingeschaltet, wenn die Optik in die Schleuse eingesetzt ist.

Cave: Xenon Kaltlicht sollte zwischen den Operationen angelasssen, nur heruntergeregelt werden. Das Starten belastet die Lampe am meisten.

Ein früheres Einschalten kann zur Erwärmung des Lichtkabelendes und damit nicht nur zum Durchschmoren der wasserdichten Abdeckung, sondern auch zu Brandverletzungen am Patientenbein führen $[3,4]$.

\section{Lichtkabel}

Unterschieden werden Fiberglas- und Fluidlichtkabel.

Die Funktion besteht in der Überleitung des von der Lichtquelle zur Verfügung gestellten Lichtes zur Optik. Prinzipiell gilt, je länger das Kabel, desto größer der Lichtenergieverlust. Einige Kabel bestehen aus Fasern, die zusammengeklebt wurden, andere, die zusammengeschweißt wurden, welches die Geschwindigkeit der Lichtübertragung steigert.

\section{Fiberglaskabel}

Diese Kabel leiten das Licht über gebündelte Glasfasern und sind daher sehr flexibel. Diese Fasern werden bei Biegung unterschiedlich starken Biegekräften ausgesetzt, sodass schon bei der normalen Handhabung Fasern brechen können. Ein signifikanter Verlust der Lichtleitfähigkeit tritt jedoch erst ein, wenn mehr als $50 \%$ der Fasern beschädigt sind. Das Brechen der Fasern stellt einen normalen Alterungsprozess dar. Deshalb ist eine Kontrolle der Lichtleitkabel in regelmäßigen Abständen notwendig. Fiberglaskabel sind günstiger in der Anschaffung als Fluidkabel und einfacher zu sterilisieren (Dampfsterilisierung). Eine Dampf-
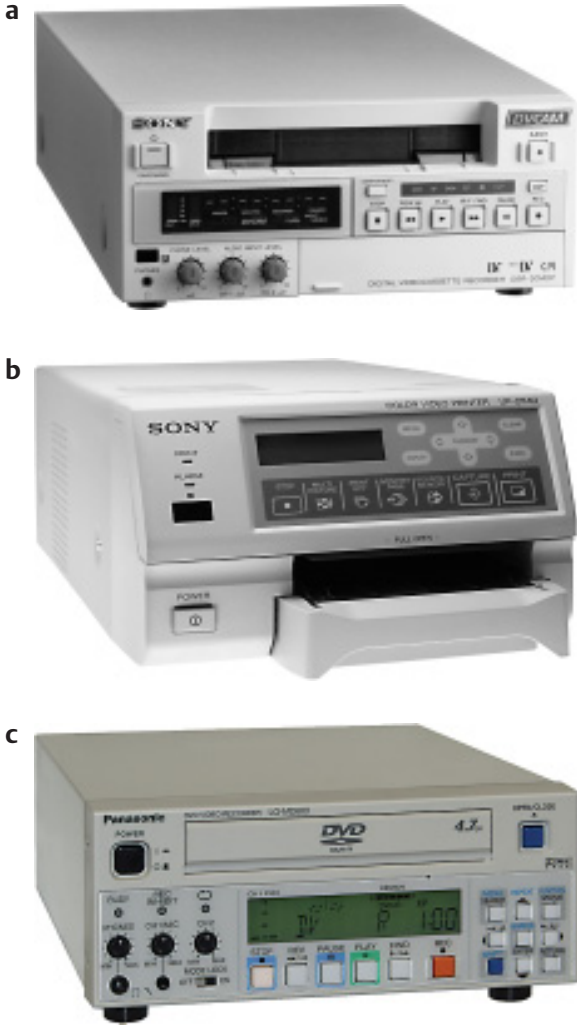

Abb. 4 Einige Möglichkeiten für Aufzeichnungsgeräte innerhalb einer Videokette. (a) Videorekorder, (b) Videoprinter, (c) DVD-Videorekorder (Fa. Wolf, Fa. Sony und Fa. Panasonic).

sterilisierung ist bei Fluidkabeln nicht möglich. Normalerweise empfiehlt sich eine Länge von $150-230 \mathrm{~cm}$, wobei längere Lichtkabel bei aufwändigeren Lagerungstechniken wie der Ellenbogenarthroskopie hilfreich sind.

Zur Dokumentation gebrochener Glasfasern des Lichtleitkabels wird dessen Ende, das normalerweise in die Lichtquelle eingesteckt wird, gegen eine Deckenlampe gehalten. Blickt man nun in das andere Ende des Lichtleitkabel zeigen sich helle und dunkle Areale, wobei die dunklen Areale dem Anteil der gebrochenen Glasfasern entsprechen.

Bei dünnen Optiken sollten dünne Kaltlichtkabel verwendet werden. Sie sind billiger und leichter. Das Bild wird besser fokussiert.

\section{Fluidkabel}

Sie bestehen aus einem mit einer speziellen Flüssigkeit gefüllten Kunststoffschlauch. Im Vergleich zu den Fiberglaskabeln ist die Lichtleitfähigkeit um 40 $50 \%$ erhöht. Die Fluidkabel sind aber wesentlich starrer und deshalb problemati-

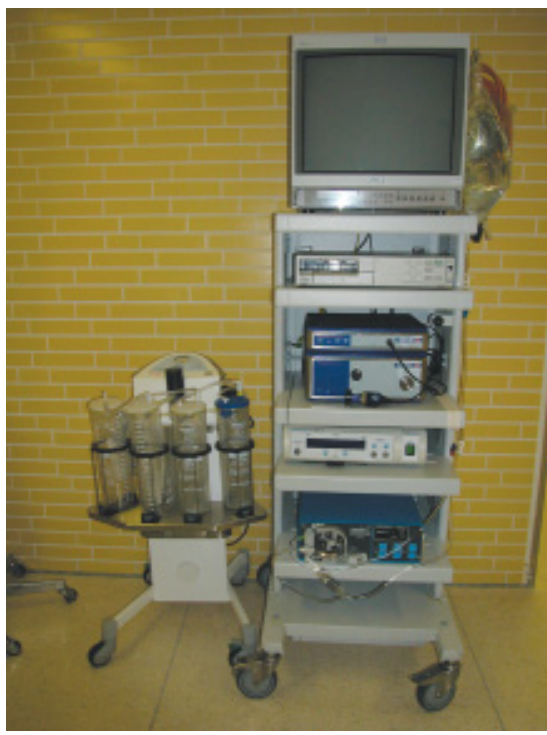

Abb.5 In einem Arthroskopieturm werden die Einzelkomponenten für die Arthroskopie zusammengefasst. Der Monitor, Videoprinter, Kaltlichtquelle und Videokamera-Steuereinheit, Shaver-Einheit, Rollenpumpe. Seitlich angebrachte Spülbeutel und externes Absaugsystem.

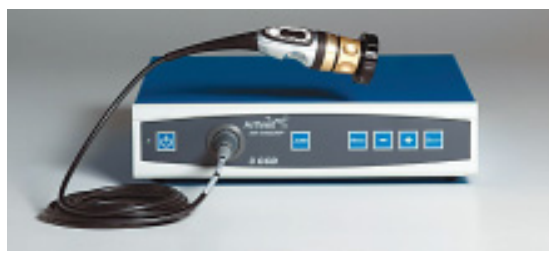

Abb. 6 Steuereinheit mit über Kamerakabel angeschlossener 3-Chip-Kamera (Fa. Arthrex).

scher zu handhaben. Wegen der empfindlichen Flüssigkeit ist eine Sterilisation durch einlegen des Lichtkabels in eine Desinfektionslösung zu bevorzugen. Die Fluidkabel bieten eine originalgetreuere Weiterleitung des Lichts und lassen die intraartikulären Strukturen eher etwas bläulich erscheinen. Dies entspricht auch eher dem natürlichen Licht des Gelenkknorpels als das weiche und wärmere Licht, das mit dem Fiberglaskabel übertragen wird. Aufgrund der Nachteile, nicht dampfsterilisierbar, empfindlich und teuer, bleiben Fluidkabel speziellen Einsätzen vorbehalten, bei denen es auf möglichst originalgetreue Wiedergabe der Gelenkstrukturen z.B. für Videoaufnahmen und hochwertigen Bilddokumentationen arthroskopischer Befunde ankommt. Die Bedeutung der Lichtkabel für eine gute Bildqualität wird vielfach unterschätzt und erst der Vergleich eines häufig gebrauchten und mit einem neuen Lichtkabel bei identischer Einstellung 

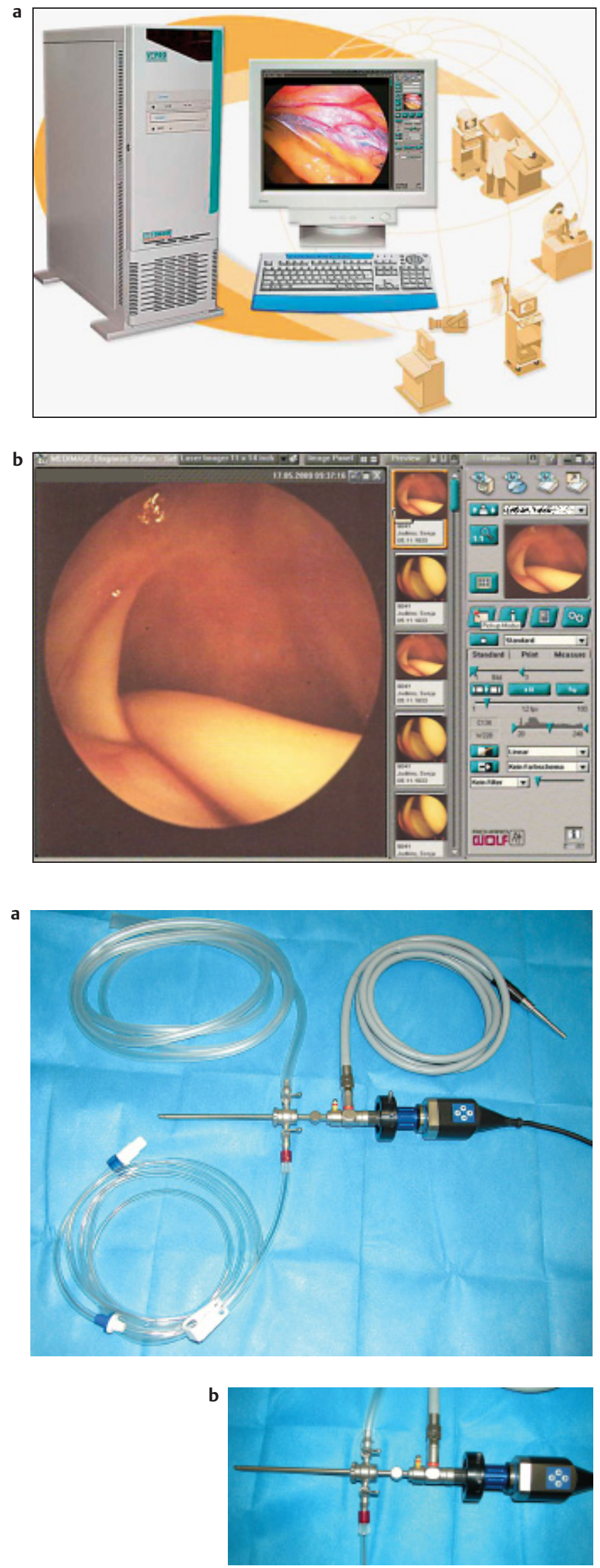

Abb. 7 (a) Beispiel einer Vernetzung zur digitalen Datenerfassung. (b) Digitale Aufbereitung von Arthroskopiebildern am Monitor ( $\mathrm{Fa}$. Wolf).

Abb. 8 Schleusensytem mit Flüssigkeitszulauf unten, Absaugschlauch oben links und angeschlossenem Kaltlichkabel oben rechts sowie eingebrachter Optik mit angeschlossener Kamera. von Lichtquelle und Kamera zeigt nicht selten eine verblüffende Aufhellung des Monitorbildes [3,4].

\section{Videokette}

Die wesentlichen Bestandteile des Videosystems sind die Videokameraeinheit und der Monitor. An die Videokette sind Aufzeichnungsgeräte wie Videorekorder, ein Videoprinter oder digitaler Standbildrekorder mit Videosequenzen anzuschließen (Abb. 4). Wichtigster Baustein der Videokette ist die Videokamera. Diese besteht wiederum aus der eigentlichen Videokamera, dem Kamerakabel und der Steuereinheit, die auf dem Arthroskopieturm platziert wird (Abb. 5).

Die anfänglichen verwendeten Röhrenkameras hatten nicht nur den Nachteil eines höheren Gewichtes und einer größeren Kamera, sondern waren vom Röhrensystem auch sehr empfindlich. Daher hat heute die Chip-Kamera die größte Verwendung. Die Einchip-Kamera besitzt einen Chip, mit dieser Kamera ist eine Auflösung von mehr als 450 Linien möglich. Mit programmierbaren Tasten am Kameragehäuse können verschiedene Kamerafunktionen oder Funktionen von Peripheriegeräten per Fingerdruck vom Operateur gesteuert werden. Mit einem Parfokalzoomsystem (KST) sind die Vorteile eines Zoomobjektivs wesentlich suffizienter zu nutzen, da hierbei ein Nachfokussieren entfällt. Ein in die Kamera integriertes digitales Bildprozessormodul erhöht die Bildqualität nochmals. Dieses Modul entspricht im wesentlichen dem Digivideo.

Bei der 3-Chip-Kamera (Abb. 6) werden die 3 Grundfarben von jeweils einem Chip getrennt erfasst und verarbeitet. Damit ist eine Farbwiedergabe sehr hoher Natürlichkeit bei gleichzeitig höchster Wiedergabequalität möglich. Durch ein sehr großes horizontales Auflösungsverhalten von über 750 Linien werden selbst feinste Gewebeunterschiede darstellbar. Neben einer digitalen automatischen Belichtungsregulierung und digitalen Farbeinstellungen weisen moderne Kameras auch einen automatischen Weißabgleich auf. Die Bildfokussierung sowie die Größe des Bildes können über den vorhandenen Parfokalzoom geregelt werden. Die neueste Entwicklung ist die digitale Kameraplattform, bei der das optische Bild bereits unmittelbar nach dem Bildchip in digitale Signale umgewandelt wird (z.B. Image $1 \mathrm{KST}$ ). Dadurch wird eine voll- 

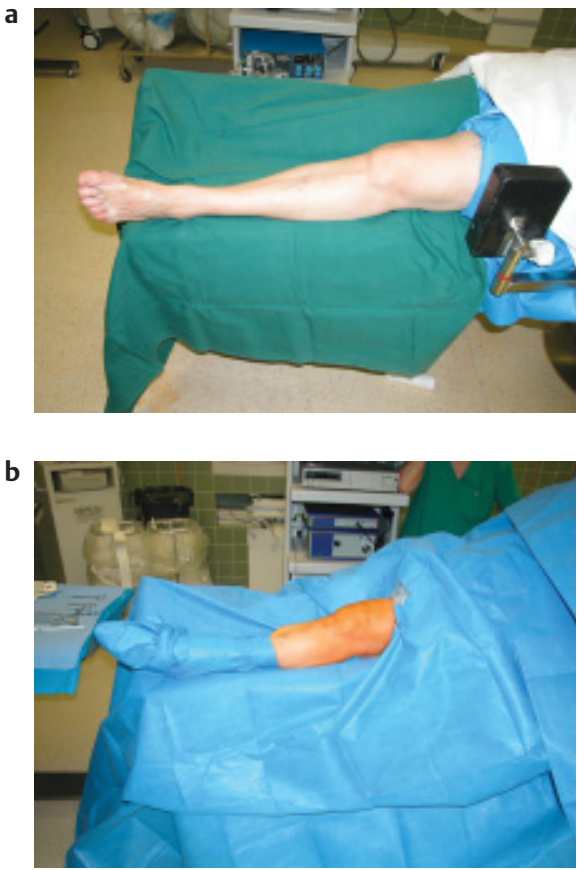

Abb.9 (a) Lagerung des Kniegelenkes mit Seitenstütze und bereits abgedeckter Oberschenkelblutsperre (b) nach steriler, wasserdichter Abdeckung.

ständig digitale Videokette - von der Bildaufnahme bis zur Bildspeicherung möglich, was sich in gleichbleibender, verlustfreier Bildqualität und einfacher und komfortabler Weiterverarbeitung niederschlägt. Während frühere Digitalkameras MOS (Metalloxydsemikonduktoren) und CPD (Charged Primary Device) Sensoren nutzten, basieren die heutigen Digitalsysteme auf dem CCD-System (Charged Coupel Device System) wegen ihrer hohen Farbsensitivität und besseren Farbwiedergabe. Die digitalen Kamerasysteme besitzen eine hohe Auflösung und erlauben die Aufbereitung der Bilder durch eine entsprechende Software und die Aufbewahrung der Daten in verschiedenen Formaten, z.B. JPEG, MPEG, BMP und TIFF (Abb. 7) [2].

\section{Steuergerät}

Das Steuergerät, an welches das Kamerakabel angeschlossen wird, bereitet die digitalisierten Signale auf und leitet sie an den Monitor. Die Steuereinheit weist Regler, z. B. für den Weißabgleich oder für die Bilddarstellung, auf. Das Kamerakabel verbindet die Kamera mit der Steuereinheit, sollte ausreichend flexibel sein und Bewegungen des Arthroskops nicht behindern. Für Einchip-bzw. Dreichipkameras sind unterschiedliche Steuergeräte notwendig.

\section{Sterilisation}

Durch Einlegen in eine Desinfektionslösung können Kameras keimarm gemacht werden. Eine $100 \%$ ige Sterilität ist jedoch nicht möglich, da die aggressiven Lösungen Kameragehäuse und Dichtungen angreifen können. Gassterilisation ist möglich, inzwischen sind autoklavierbare Kameras erhältlich. Deshalb wird die Kamera präoperativ in einen sterilen Plastikschlauch aus Einmalmaterial eingepackt. Dieser wird zunächst von der Operationsschwester mit feinen Klebebändern fest am Okular der Optik fixiert. In den sterilen Plastikschlauch wird dann die unsterile Kamera eingeführt, der Außenteil des Schlauches bleibt dabei steril. Mit diesem einfachen Hilfsmittel wird die Sterilität während der arthroskopischen Operationen eingehalten.

\section{Monitor}

Der Monitor sollte ausreichend groß gewählt werden, da oft sehr feine Details aus dem Gelenk dargestellt werden. $\mathrm{Zu}$ empfehlen ist ein Monitor mit einer 51cm-Bildröhre bzw. Flatscreen, die in $\mathrm{Zu}-$ kunft nur noch erhältlich sein werden.

\section{Digivideo}

Um einen besseren Kontrast zu gewährleisten, wurde ein digitales Bildverarbeitungssystem entwickelt, dass eine digitale Kontrast- und Schärfenverbesserung des Videobildes in Echtzeit durchführt. Dabei wird zunächst das gesamte Videobild digitalisiert und digital weiter verarbeitet. Die digitalen Werte werden dann nach definierten Zielkriterien einer zweidimensonalen Filterung unterzogen und gewichtet. Die Gewichtsfaktoren werden dabei so ausgewählt, dass eine für das visuelle Empfinden des Menschen deutlich erhöhte Kontrast- und Schärfenanhebung erzielt werden kann. Um diese Form der Bildverarbeitung in Echtzeit zu ermöglichen, sind etwa 50 Mill. Rechenschritte pro Sekunde notwendig. Ein entsprechender Mikroprozessor ist daher im System integriert. In alle Einchipund Dreichip-Kameras ist bereits ein digitales Bildprozessorsystem integriert.

\section{Twinvideo}

Das Twinvideo (KST) ist ein digitaler Bildim-Bild-Prozessor, der 2 verschiedene Bildinformationen gleichzeitig auf dem Monitor darstellen kann. Um einen präzisen Vergleich zu ermöglichen, können parallel zum Operationsgeschehen Bilder
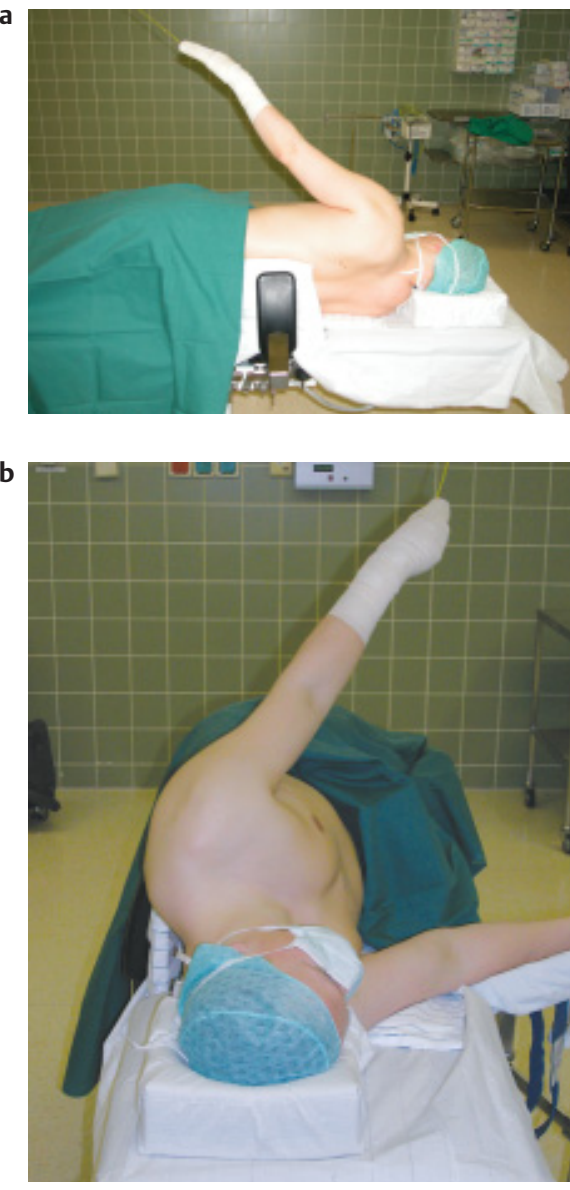

Abb.10 Seitenlagerung für die Schulterarthroskopie. (a)Von dorsal: Extension der Schulter über einen Unterarmhalter in ca. $45^{\circ}$-Abduktion mit 4-6 kg. 30'-Kippung des Oberkörpers in der Achse nach hinten. (b) Von kranial: Anteversion von ca. $25^{\circ}$ der Schulter.

vom Videorekorder von einem CD-Player oder sonstige digitale Bildinformationen von vorausgegangenen Operationen oder Vorbefunden eingespielt werden. Dieses System ist besonders hilfreich bei Bildübertragungen aus dem Operationssaal.

\section{Auffüllmedium}

Im Normalzustand befinden sich nur wenige Milliliter Synovialflüssigkeit im Kniegelenk. Da eine Inspektion unter diesen Bedingungen nicht möglich ist, muss das Gelenk aufgefüllt werden.

Man unterscheidet ein gasförmiges von einem flüssigen Auffüllmedium. Während früher noch mit Kohlendioxyd und Luft als gasförmige Auffüllmedien gearbeitet wurde, ist dieses Vorgehen heutzutage zugunsten der flüssigen Auffüllmedien weitgehend verlassen 
worden. Als Flüssigkeiten werden Ringerlactat, isotonische Kochsalzlösung und elektrolytfreie Lösung verwendet.

Wird elektrochirurgisch gearbeitet, ist eine elektrolytfreie Lösung notwendig.

\section{Spülsystem}

Bei der Arthroskopie im flüssigen Milieu muss das Auffüllmedium in das Gelenk gebracht und auch wieder aus dem Gelenk entfernt werden. Für Flüssigkeitszulauf in das Gelenk wird ein steriles Schlauchsystem, welches zum einen am Flüssigkeitsbeutel und zum andern an einem Hahn der Schleuse angeschlossen wird, benützt (Abb. 8). Hierbei kann der erforderliche Füllungsdruck auf 2 verschiedene Arten erzeugt werden:

1. Über ein Pumpensystem (Rollenpumpe) - hierzu ist eine spezielle Rollenpumpe notwendig, in die der Zulaufschlauch eingelegt wird. Pumpen arbeiten druck- oder volumengesteuert. Der maximal erreichbare Füllungsdruck kann definiert eingestellt werden. Druckgesteuerte Rollenpumpen haben den Nachteil, des nicht unbeträchtlichen Anschaffungspreises.

2. Wesentlich günstiger ist dabei Auffüllung mittels Schwerkraft. Dabei wird durch Höher- bzw. Tieferhängen der Flüssigkeitsbeutel der Auffülldruck gesteigert bzw. reduziert. Hängen die Flüssigkeitsbeutel etwa 1,20 bzw. $1,50 \mathrm{~m}$ über dem Knieniveau, ist ein ausreichender Füllungsdruck gegeben.

\section{Lagerung}

Kniegelenk

Die Lagerung mit gestrecktem Bein erfüllt alle Anforderungen, die an eine einfache, schnelle und sichere Lagerungstechnik zu stellen sind. Auf der Lateralseite des Oberschenkels wird eine einfache Seitenstütze am OP-Tisch angebracht (Abb. 9). Die Stütze wird etwa handbreit proximal der Patellabasis positioniert.

Ein Vorteil dieser Lagerung liegt darin, dass der Operateur bei der Arthroskopie sitzen kann. Fehlermöglichkeiten bestehen in der zu weit distalen Positionierung der Seitenstützen, sodass Anlage des Arthroskopzugangs und Bewegung des Arthroskops behindert werden. Auch die Lagerung im Kniegelenk kann erschwert sein. Bei einer zu weit proximalen Position der Seitenstütze ist die mediale Aufklappbarkeit zur Inspektion der Innenmeniskushinterhorns er-

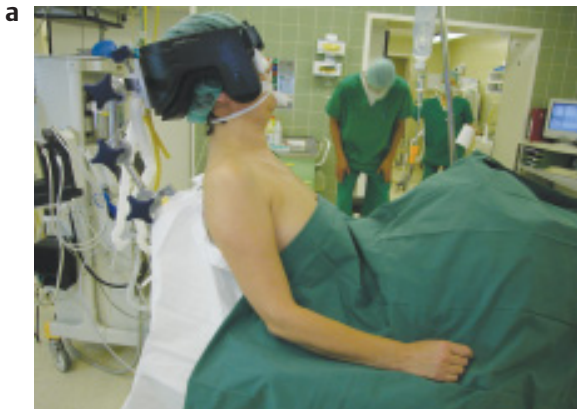

lenk bewährt [1]. Die Seitenlagerung ist aber auch für die Rekonstruktion der Rotatorenmanschette über Miniarthrotomie oder eine Bizepssehnentendodese gut geeignet.

Besonders zu beachten ist dass sorgfältige Abpolstern knöcherner Vorsprünge (Ellenbogen, Knie, Fibulaköpfchen, Sprunggelenke), um entsprechende Lagerungsschäden zu vermeiden.
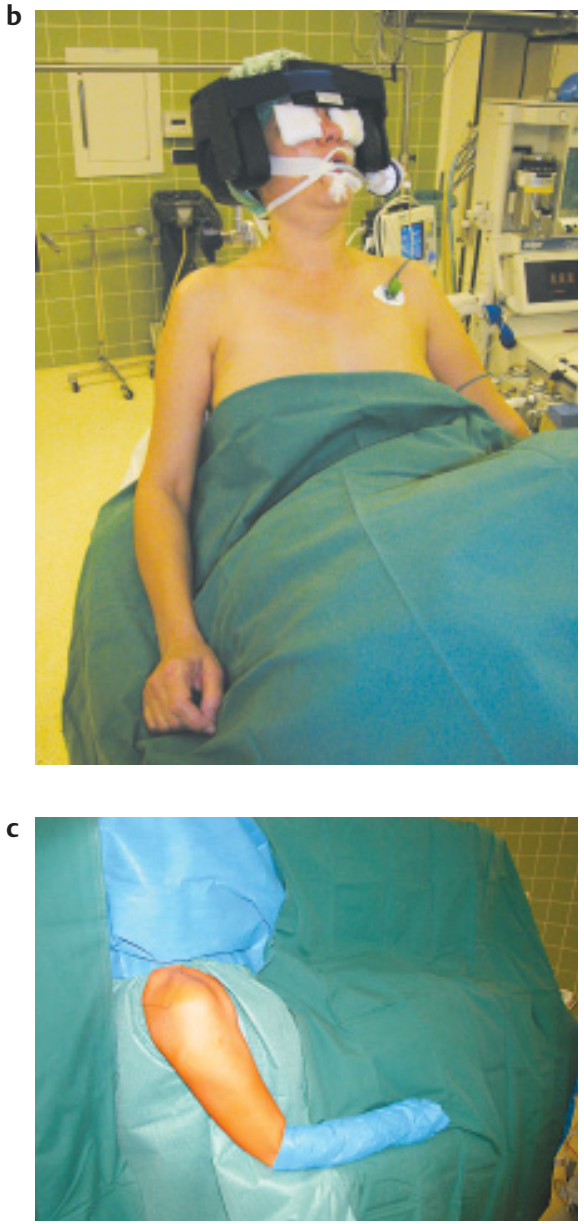

Abb.11 Beach-chair-Lagerung. (a) Von lateral: ca. $70^{\circ}$ Oberkörperhochlagerung, (b) von kaudal. (c) Nach steriler, wasserdichter Abdeckung.

schwert, da der Oberschenkel aufgrund des vermehrten Weichteilmantels im proximalen Anteil nicht so gut gegen die Seitenstütze gedrückt werden kann.

\section{Schulter}

\section{Seitenlagerung}

Die Seitenlagerung des Patienten (Abb. 10) hat sich für arthroskopische Eingriffe im glenohumoralen Gelenk, subakromialen Raum und am Akromioklavikularge-
Der Patient sollte mit dem Rücken direkt an der Tischkante gelagert werden, sodass sich der dorsal von ihm stehende Operateur bei Manipulationen an den ventralen Zugängen nicht so weit nach vorne überneigen muss. Der Rumpf wird über gepolsterte Gliederstützen ventral an der unteren Thoraxapertur und dorsal auf Höhe der Beckenkämme fixiert. Unter den Thorax wird eine Tuchrolle gelegt, um die Axilla zu entlasten und Schäden am Plexus brachialis zu verhindern. Der Kopf soll nicht abgeknickt sein, er wird in einem Schaumstoffpolster so gelagert, dass HWS und BWS eine Linie bilden. Die Seitlagerung wird noch dahin korrigiert, dass der Oberkörper des Patienten um $30^{\circ}$ um die Längsachse nach hinten gedreht wird. Hierdurch stellt sich die Pfannenebene der Schulter parallel zum Operationstisch ein. Die Instrumentation von ventral wird erleichtert, sodass sich die Operationsinstrumente nicht in der ventralen Abdeckung verfangen können. An Unterarm und Handgelenk wird eine vorgefertigte Unterarmgamasche mit Polsterung angewickelt, um den Arm am Schulterhalter zu befestigen. Dies ist unerlässlich, um bei der Arthroskopie den Arm in alle gewünschten Positionen zu bringen und für die Distraktion konstante Bedingungen zu schaffen, damit bei der intraartikulären Manipulation die Gelenkflächen nicht iatrogen geschädigt werden.

Der Arm wird im Schulterhalter bei ca. $45^{\circ}$-Abduktion und $25^{\circ}$-Anteversion unter 4-6 kg Zuggewicht gelagert. Je nach Bedarf kann der Abduktionswinkel vergrößert oder verringert werden. Um Traktionsschäden am Plexus brachialis zu vermeiden, sollte die Abduktion nicht über längere Zeit $45^{\circ}$ überschreiten. Aus demselben Grund sollte je nach Konstitution des Patienten das Traktionsgewicht 4-6 kg nicht überschreiten.

Für rekonstruktive Eingriffe am LabrumLigament-Komplex ist ein Armhalter mit einer doppelten Zugfunktion hilfreich. Mit ihm ist eine kontinuierliche Distrak- 

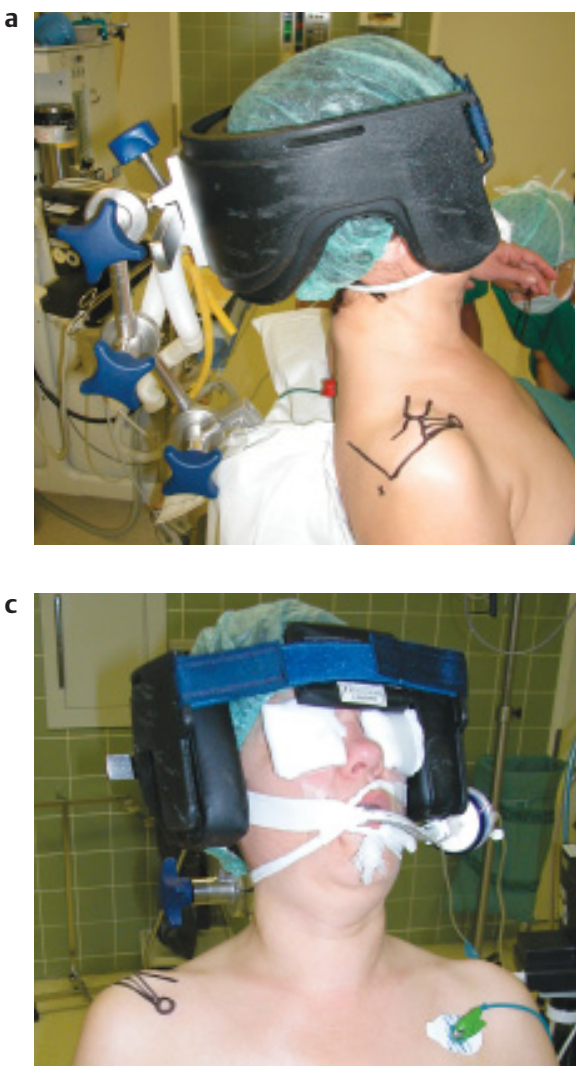

tion des glenohumoralen Gelenks ohne Dislokation des Humeruskopfes möglich.

\section{Beach-chair-Lagerung}

Alternativ kann die halbsitzende Beachchair-Position gewählt werden, wenn im Anschluss an einen arthroskopischen Eingriff eine offene Rekonstruktion der Rotatorenmanschette oder der ventralen Kapsel geplant ist [1].

Diese Lagerung ist weniger aufwändig und für die Anästhesie angenehmer, da der Zugang zu den Atemwegen günstiger ist (Abb. 11). Für die Beach-Chair-Lagerung wird die Rückenstütze des OP-Tisches so eingestellt, dass der Oberkörper des Patienten ca. $70^{\circ}$ zur Horizontalen bildet. Für die Lagerung des Kopfes verwenden wir eine spezielle Lagerungsschale, die als Verlängerung am OP-Tisch angebracht ist und sich nach lateral zur Seite der betroffenen Schulter verschieben lässt. Der Oberkörper des Patienten wird weit lateral positioniert, sodass die Schulter von ventral und dorsal frei zugänglich ist. Alternativ sind spezielle OP-Tische mit abnehmbarem dorsolateralen Schulterpolster vorhanden. Der Arm des Patienten hängt frei zur Seite. Sehr hilfreich ist ein Lagerungskissen unter der medialen Skapula, um die Schulter nach vorne zu

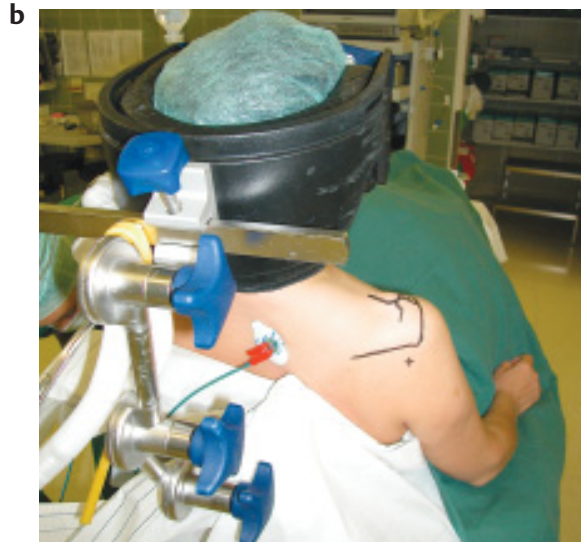

Abb.12 Kopfhalterung für die Beach-ChairLagerung mit eingezeichneten anatomischen Landmarken für die Schulterarthroskopie und dem dorsalen Portal (x). Von (a) lateral, (b) dorsal mit bereits entfernten dorsolateralem Tischpolster, (c) anterior mit Fixierung des Kopfes durch Klettverschlussband.

bringen. Zur Sicherung des Patienten wird ein Beckengurt und eine Seitenstütze auf Thoraxhöhe verwendet. Kopf und Halswirbelsäule werden in einer möglichst neutralen Position eingestellt. Der Kopf wird dann mit einem vorgegebenen Klettverschluss in Stirnhöhe an der Lagerungsschale fixiert (Abb. 12).

\section{Dokumentation}

Die wichtigste und bewährteste Dokumentationsform ist nach wie vor der Operationsbericht. Jede Naturbetrachtung trägt nur dann Früchte, wenn sie dem Nächsten mitgeteilt wird. Schon Isaac Newton konnte sich nicht darauf beschränken, eine Gravitationslehre abzuleiten und zu beobachten, wie Äpfel zu Boden fallen. Er musste alles auch aufschreiben und dem Royal College vortragen. Beobachtung und Beschreibung eines naturwissenschaftlichen Tatbestandes gehören untrennbar zusammen. Im Operationsbericht werden folgende Punkte berücksichtigt: kurze Anamnese, klinischer Untersuchungsbefund, z.B. Jerk-Test, Narkoseuntersuchung, Lagerungsparameter, wie Rückenlage, BeachChair-Position etc., Operation in Blutsperre, Arthroskopiezugang, Beschaffenheit des Auffüllmediums und schließlich systematischer Untersuchungsgang, wobei normale und pathologische Befunde aufgeführt werden (retropatellarer Knorpel, oberer Rezessus, lateraler Rezessus, Lateralisation der Patella, mediales Gelenkkompartment mit dem entsprechenden Knorpelzustand und Innenmeniskus, Area intercondylaris mit vorderem
Kreuzband und hinterem Kreuzband, laterales Gelenkkompartment mit Außenmeniskus, Hiatus popliteus und dorsolateralem Rezessus). Es wird dann der Instrumentenzugang erwähnt und die durchgeführten operativen Maßnahmen beschrieben. Beendigung der Arthroskopie mit Einlegen einer Redondrainage, postoperatives Prozedere.

\section{Dokumentationsbögen}

Ein schneller Informationsfluss ist möglich über standardisierte Befundbögen, in denen die wichtigsten Punkte angekreuzt werden und mithilfe von Schemazeichnungen noch entsprechend präzisiert werden. Neben diesen beiden Möglichkeiten bildet die Bilddokumentation natürlich eine sehr gute Ergänzung. Die heute am meisten verbreitete Bilddokumentation erfolgt mit dem Videoprinter. Diese Dokumentationsform ist einfach, schnell, preisgünstig und erlaubt eine einfach Archivierung in der Patientenakte. Die mitgegebenen Bilder werden vom Patienten sehr positiv bewertet, darüber hinaus ist ein hoher Informationsgehalt gegeben.

\section{Digitaler Standbildrekorder}

Mit dieser Technologie ist die digitale Speicherung und Archivierung der Bildinformation möglich. Der Standbildrekorder gewährleistet die Anforderung einer Einfrierfunktion des Videobildes und die Abspeicherung des Bildes als Datei auf einem Datenträger.

\section{Literatur}

1 Habermeyer P. Schulterchirurgie Urban und Fischer, München-Jena 2002

2 Lajtai G, Snyder SJ, Appelgate GR, Aitzetmüller G, Gerber G. Shoulder Arthroscopy and MRI Techniques. Springer Verlag Berlin-Heidelberg-New York 2003

3 Strobel M. Arthroskopische Chirurgie. Springer Verlag Berlin-Heidelberg-New York 1998

4 Strobel M, Eichhorn J, Schiessler W. Arthroskopie des Kniegelenkes, Grundprinzipien Diagnostische Arthroskopie, Arthroskopische Chirurgie. Deutscher Ärzteverlag Köln 1996

\section{Dr. med. Werner Koithan}

Funktionsoberarzt

Klinik für Unfall- und Wieder-

herstellungschirurgie

Allgemeines Krankenhaus

Siemensplatz 4

D-29223 Celle 